\title{
Unique Presentation of Methamphetamine- Induced Cardiomyopathy with Multiple Bilateral Pulmonary Emboli
}

\author{
Javad Najjar Mojarrab ${ }^{1 *}$, Somto Nwaedozie ${ }^{1}$ and Ali Al-Hilli ${ }^{3}$ \\ ${ }^{1}$ Department of Internal Medicine, Marshfield Clinic Health System (MCHS) - Marshfield, 1000 North Oak Avenue 3K2, Marshfield, WI 54449, USA
}

${ }^{2}$ Department of Internal Medicine, MCHS - Marshfield, 1000 North Oak Avenue 3K2, Marshfield, WI 54449, USA

${ }^{3}$ Department of Hospital Medicine, MCHS - Marshfield, 1000 North Oak Avenue 3N, Marshfield, WI 54449, USA

Submission: March 10, 2021; Published: March 16, 2021

*Corresponding author: Javad Najjar Mojarrab, MD, MBA; Marshfield Clinic Health System (MCHS)-Marshfield, Department of Internal Medicine, 1000 North Oak Avenue 3K2, Marshfield, WI 54449, USA

\begin{abstract}
Methamphetamine is an amphetamine type stimulant that adversely impacts multiple organ systems and is a growing public health problem worldwide. We present a case of a 35-year-old farmer with no previous cardiac history, yet significant history of methamphetamine abuse who experienced worsening shortness of breath, productive cough with hemoptysis, bilateral leg swelling, and orthopnea. Initial laboratory testing were remarkable for mildly elevated levels of troponin, C-reactive protein, beta natriuretic peptide, and D-dimer. Chest radiography revealed cardiomegaly and a small, right-sided pleural effusion. Further diagnostic imaging by computed tomography angiography highlighted bilateral sub-segmental pulmonary embolism (PE) while cardiac echocardiography detected severely dilated cardiomyopathy of all chambers with severely reduced ejection fraction (EF). Additional cardiac and hematological workups were negative for ischemic disease, infection, or thromboembolic disease. The patient was diagnosed with methamphetamine-associated cardiomyopathy (MAC) and PE and treated for new onset heart failure with reduced ejection fraction. Though symptoms improved with cessation of drug use, follow up imaging at two years revealed sustained cardiac damage. MAC can rarely present in association with PE in patients with a history of methamphetamine abuse. A high index of clinical suspicion is necessary to identify this life-threatening combination in young adults.
\end{abstract}

Keywords: Methamphetamine-associated cardiomyopathy; MAC; Pulmonary embolism; PE; Deep vein thrombosis; DVT

\section{Learning Objective}

Methamphetamine use is associated with cardiac pathologies. Development of disseminated pulmonary embolism and severely reduced cardiac ejection fraction in the setting of polysubstance abuse in an adult patient $<40$ years of age is a rare presentation of methamphetamine-associated cardiomyopathy. Additional diagnostic workup for pulmonary embolism and cardiomyopathy is warranted for young adult patients with a history of methamphetamine use and thromboembolic events who experience constant dyspnea and rapid weight gain over a period of weeks.

\section{Introduction}

Methamphetamine use has been growing steadily in the United States since the 1990s, and is the second most abused class of recreational drugs worldwide [1]. Methamphetamine use is associated with a wide range of psychosocial and psychophysical health problems as well as early mortality [1-9]. Cardiovascular problems caused by methamphetamine use have been well described, and among them are malignant hypertension, arrhythmia, aortic dissection, myocardial infarction, and cardiomyopathy [2-10]. Adverse cardiovascular effects are due to the excess presence of neurotransmitters, mainly norepinephrine, in the circulatory system [10]. We present a case of dilated cardiomyopathy in a young male patient with significant history of methamphetamine abuse.

\section{Case Report}

A 35-year-old male farmer with past medical history significant for asthma, history of deep vein thrombosis (DVT) treated with warfarin for 6 months, and polysubstance abuse was brought into the emergency department complaining of increasing shortness of breath, bilateral leg swelling, and a $9.07 \mathrm{~kg}(20 \mathrm{lb})$ weight gain for 2 weeks. He also complained of orthopnea and paroxysmal nocturnal dyspnea for 2 weeks. A few days before his admission, he also experienced right-sided pleuritic chest pain rated at an 
8 out 10 in intensity, productive cough, and hemoptysis. The patient is a smoker and admitted to recreational drug use, notably intravenous (IV) methamphetamine twice daily for one year before his presentation, but he denied alcohol use. His family history is significant for history of DVT in his mother. On examination, the patient appeared anxious and in mild respiratory distress with oxygen saturation of mid-90s on room air. His was tachycardic with a heart rate in the range of 120 s to 130 s (Figure 1). He had bilateral crackles on respiratory examination and $2+$ bilateral pedal edema. Initial laboratory testing revealed mildly elevated levels of cardiac biomarkers troponin (83 ng/L), C-reactive protein (CRP) (3.3 mg/dL), beta natriuretic peptide (BNP) (1,203 pg/mL), and D-dimer (2.33 mg/ L), yet normal thyroid function. His drug screen was positive for methamphetamines and oxycodone. Based on the patient's presenting signs, symptoms, and positive Well's criteria score of more than six, further tests were undertaken. A chest radiograph revealed cardiomegaly, a small, right sided pleural effusion, and peribronchial thickening or infiltrate that could be peribronchial pneumonia or atelectasis (Figure 2). Electrocardiogram (EKG) analysis of heart rhythm detected sinus tachycardia with right atrial enlargement, extreme rightsided axis deviation, and other nonspecific ST segment changes. Computed tomography angiography (CTA) highlighted bilateral sub-segmental pulmonary embolism (PE) and an enlarged, leftshifted heart. Further cardiac analysis by echocardiogram revealed severely dilated cardiomyopathy with a reduced ejection fraction (EF) of 10\% (Figure 3). 2D echocardiogram specifically revealed biventricular and atrial enlargement and severe left ventricular dysfunction, with an EF of $10 \%$ without any structural valvular lesions or wall motion abnormalities. His coronary angiogram was negative for obstructive atherosclerotic coronary artery disease. Diagnostic cardiac catheterization was also performed and revealed no coronary artery disease and was negative for ischemic disease. Guideline-directed medical therapies were initiated.

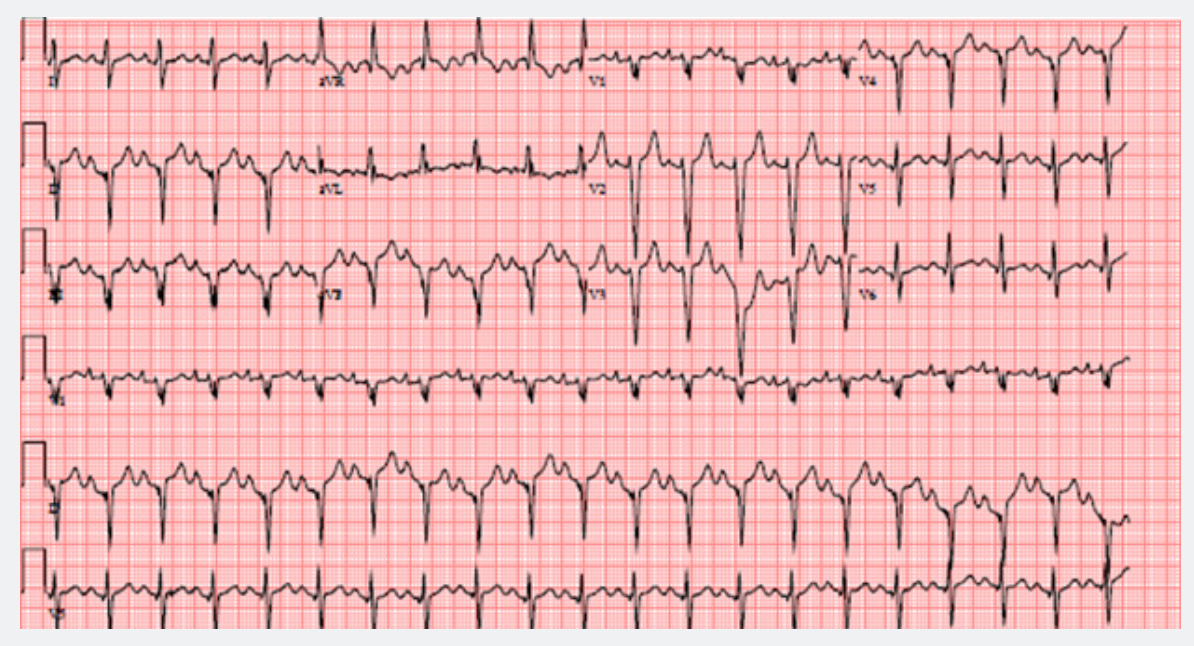

Figure 1: Electrocardiogram of sinus rhythm abnormalities.

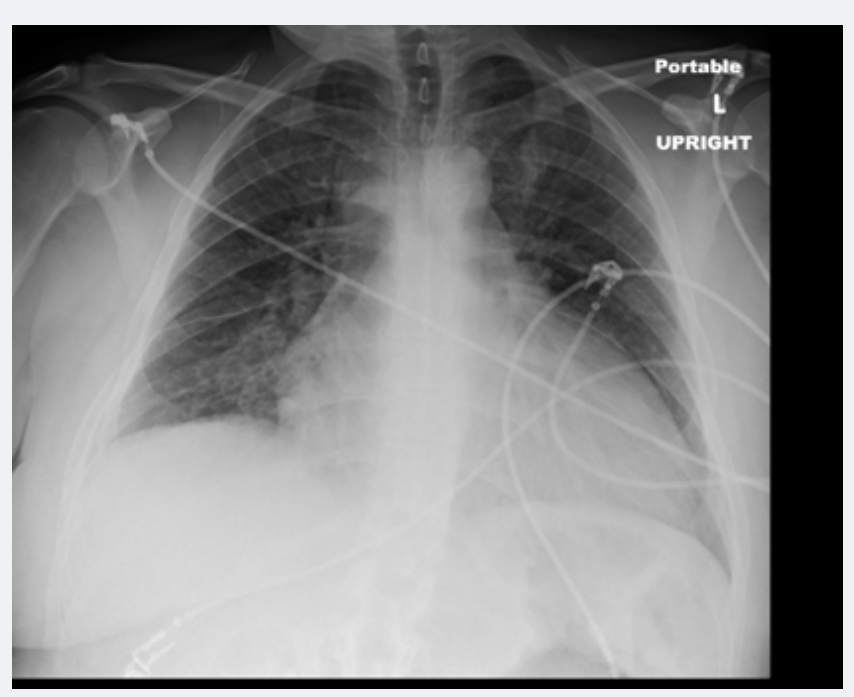

Figure 2: Chest radiograph revealing cardiomegaly and interstitial prominence. 


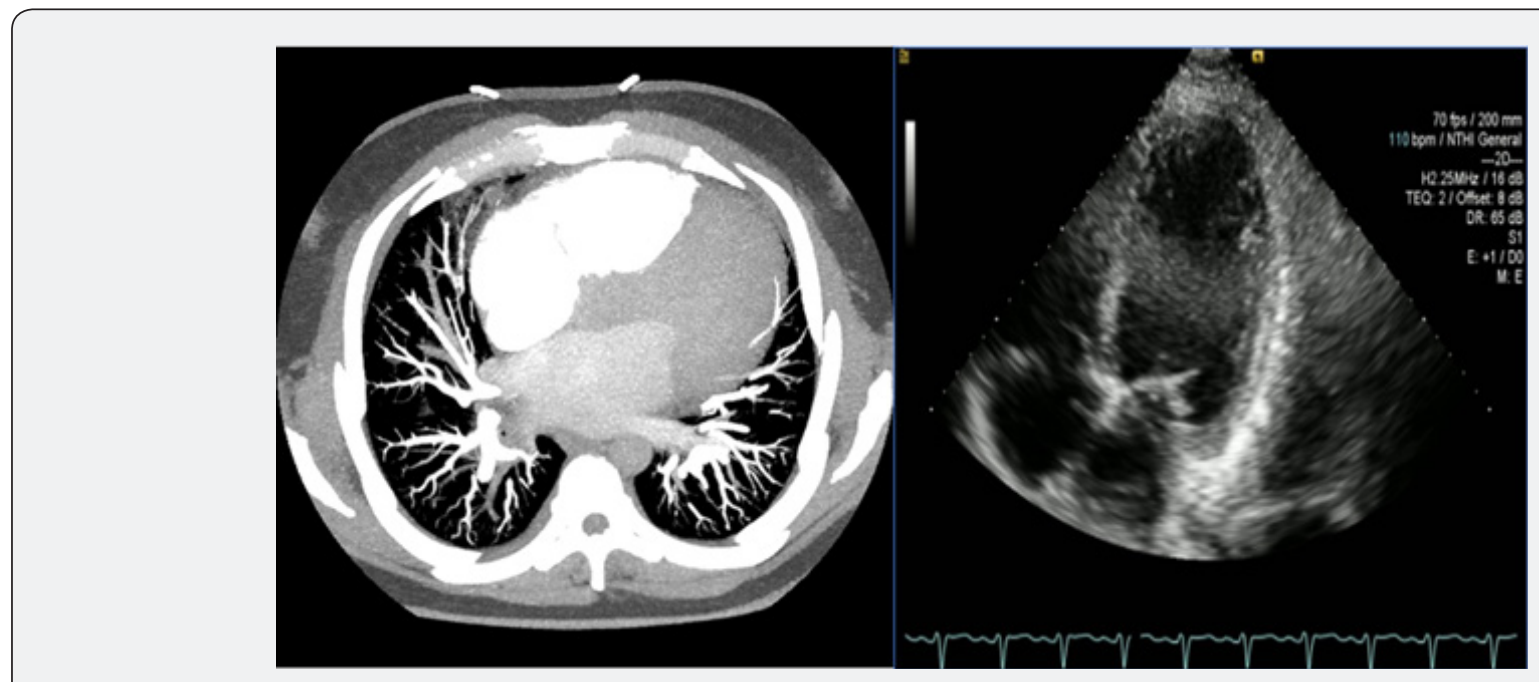

Figure 3: $[A]$ Computed tomography angiography $(C T A)$ and $[B]$ echocardiogram showing a severely dilated left ventricle.

Due to the patient's previous history of DVT, family history of DVT, and history of IV drug use, further work-up was performed to identify the underlying cause of his PE. Hypercoagulable work-up was negative for homocysteine, antiphospholipid antibodies, and cardiolipin antibodies, and his protein $\mathrm{C}$, protein S, and antithrombin III activity levels were within normal limits. Sequencing of the prothrombin gene revealed no mutations. Laboratory analysis of blood samples for pericarditis and myocarditis was also negative. Doppler ultrasound of all extremities did not reveal DVT. Though the underlying cause of the patient's PE was unknown, to prevent further PE development, the patient was started on an anticoagulant regimen of IV heparin. Though the patient's symptoms of congestive heart failure improved in the hospital, his cardiac EF was still dramatically reduced, and he was discharged home on Life Vest with a planned follow-up with cardiology. He was also placed on a prophylactic regimen of rivaroxaban for his bilateral PE. Over 2 years of followup, the patient had allegedly ceased illicit drug use and reported improved symptoms. However, repeat 2D echocardiogram analysis at 2 years revealed a sustained reduced EF of $10 \%$.

\section{Discussion}

Besides cannabis, methamphetamines are the second most commonly abused recreational substances worldwide with cardiovascular complications the leading cause of mortality among users [1-8]. Methamphetamine-associated cardiomyopathy (MAC) increased from $1.8 \%$ to $5.6 \%$ from $2009-2014$, and almost $72 \%$ of methamphetamine users exhibit at least one electrocardiogram abnormality including right axis deviation, left ventricular hypertrophy, tachyarrhythmias, and/or QTc prolongation [1,3-8].

Multifactorial mechanisms have been proposed regarding the pathogenesis of MAC including catecholamine excess, coronary vasospasm resulting in ischemia, increasing reactive oxygen species, mitochondrial injury, alterations in cardiac metabolism, and direct cardio-toxic effects [1,3-10]. These mechanisms result in observed pathologies such as ventricular hypertrophy and dilatation, fibrosis, and contraction band necrosis, which vary depending on degree and chronicity of drug use $[3,9,10]$.

Our patient's clinical symptoms and signs of acute decompensated heart failure and significantly elevated BNP of $1,203 \mathrm{pg} / \mathrm{mL}$ (normal range: $0-80 \mathrm{pg} / \mathrm{mL}$ ) at the relatively young age of 35 made us consider precipitating etiological factors like congenital heart disease, hypertensive emergency, acute coronary syndrome, cardiac arrhythmia, hyperthyroidism, myocarditis, or pericarditis, in addition to toxin-mediated cardiomyopathy. However, he had no past medical history of congenital heart disease, cardiac arrhythmia, hypertension, or endocrinopathy, and his family history was negative for cardiac disease or sudden cardiac death. He reported no preceding viral infection, associated fever, chills, or malaise, and his blood counts were unremarkable for leukocytosis or other laboratory indications of infection. His thyroid function was normal and his troponin I was minimally elevated, which excluded an endocrine disorder for his cardiac symptoms. An EKG showed sinus tachycardia with right atrial enlargement and right superior axis deviation with nonspecific ST segment changes, and continuous telemetry monitoring did not reveal any concerning arrhythmia. Additional cardiac imaging, catheterization, and hematological analysis did not reveal any structural abnormalities, obstructions, coronary artery disease, or a hypercoagulable state. This constellation of epidemiological and clinical information supported our diagnosis of a non-ischemic dilated cardiomyopathy secondary to methamphetamine abuse.

Due to his progressive shortness of breath, pleuritic chest pain, and hemoptysis, a chest computed tomographic angiography was done, which showed multiple central and peripheral bilateral pulmonary emboli. Further evaluation for the source of the emboli with Doppler ultrasound of all extremities did not reveal any 
deep or superficial venous thrombi. As a result, the pulmonary emboli were presumed to likely originate from a cardiac source predisposed by his significant biventricular enlargement and hypokinesis. Given that the patient was an ambulatory young man with low risk factors for DVT/PE, his presentation with bilateral $\mathrm{PE}$ in the setting of amphetamine-associated cardiomyopathy is exceedingly rare, and only one other case has been documented in the literature to the best of our knowledge [10].

Like other stress-induced cardiomyopathy, MAC can oftentimes be reversible with discontinuation of the offending drug alongside guideline-directed medical management with medications that modify the neurohumoral system like angiotensin-converting enzyme (ACE) inhibitor/angiotensin receptor blockers (ARBs). However, factors that impact the amount of reversal include the degree and chronicity of toxic exposure, extent of cardiac remodeling, and the time of intervention [5$7,10]$. Our patient had a high degree of IV drug exposure for over a year as well as significant cardiac remodeling at presentation. Although he received optimal medical management with prompt anticoagulation, diuretics, ACE inhibitors, and beta-blockers, demonstrated improved symptoms upon cessation of drug use, and averted hospitalization over 2 years of follow-up, repeat 2D echocardiography showed persistent biventricular dilatation and unchanged left ventricle EF of $10 \%$, indicative of irreversible cardiac damage. Prompt identification and treatment of cardiac symptoms with corresponding cessation of illicit drug use is helpful for improving quality of life in patients with a history of methamphetamine use, but this may not completely restore cardiac function to normal levels.

\section{Acknowledgements}

The authors acknowledge Emily Andreae, PhD and Marie Fleisner, MWC from the Marshfield Clinic Research Institute for manuscript editing assistance.

\section{Conflict of Interest Disclosure}

The authors declare that there are no conflicts of interest.

\section{References}

1. Durell TM, Kroutil LA, Crits-Christoph P, Barchha N, Van Brunt DL (2008) Prevalence of nonmedical methamphetamine use in the United States. Subst Abuse Treat Prev Policy 3: 19.

2. Kuo CJ, Liao YT, Chen WJ, Tsai SY, Lin SK, et al. (2011) Causes of death of patients with methamphetamine dependence: A record-linkage study. Drug Alcohol Rev 30(6): 621-628.

3. Voskoboinik A, Ihle JF, Bloom JE, Kaye DM (2016) Methamphetamineassociated cardiomyopathy: Patterns and predictors of recovery. Intern Med J 46(6): 723-727.

4. Zhao SX, Seng S, Deluna A, Yu EC, Crawford MH (2020) Comparison of clinical characteristics and outcomes of patients with reversible versus persistent methamphetamine-associated cardiomyopathy. Am J Cardiol 125(1): 127-134.

5. Won S, Hong RA, Shohet RV, Seto TB, Parikh NI (2013) Methamphetamine-associated cardiomyopathy. Clin Cardiol 36(12): 737-742.

6. Sliman S, Waalen J, Shaw D (2016) Methamphetamine-associated congestive heart failure: Increasing prevalence and relationship of clinical outcomes to continued use or abstinence. Cardiovasc Toxicol 16(4): 381-389.

7. Paratz ED, Zhao J, Sherwen AK, Scarlato RM, Macisaac AI (2017) Is an abnormal ECG just the tip of the ICE-berg? Examining the utility of electrocardiography in detecting methamphetamine-induced cardiac pathology. Heart Lung Circ 26(7): 684-689.

8. Kaye S, McKetin R (2005) National Drug and Alcohol Research Centre (Australia). Cardiotoxicity associated with methamphetamine use and signs of cardiovascular pathology among methamphetamine users. Sydney: NDARC.

9. Lord KC, Shenouda SK, McIlwain E, Charalampidis D, Lucchesi PA, et al. (2010) Oxidative stress contributes to methamphetamine-induced left ventricular dysfunction. Cardiovasc Res 87(1): 111-118.

10. Janardhanan R, Kannan A (2016) Methamphetamine cardiotoxicity: Unique presentation with multiple bi-ventricular thrombi. Am J Med 129(1): e3-e4.

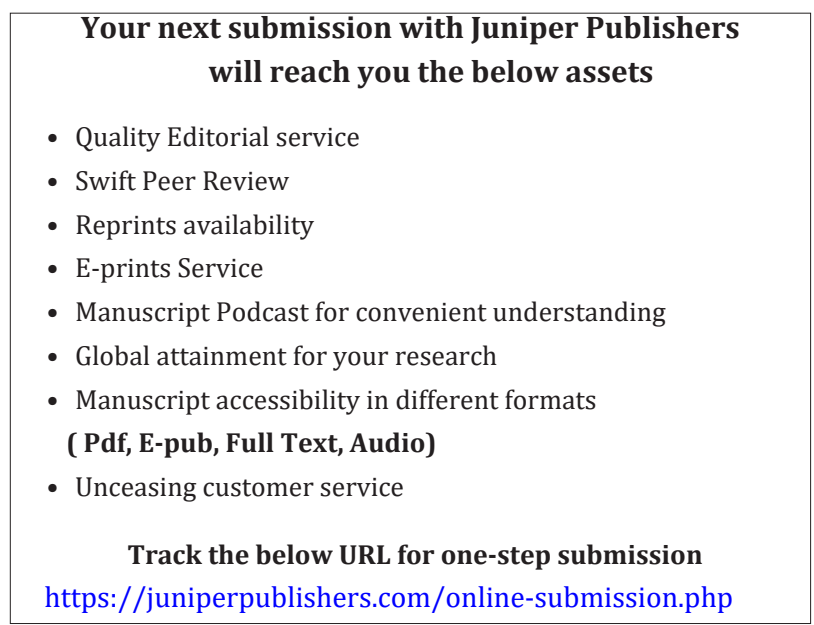

